\title{
Can one improve the Froissart bound? ${ }^{12}$
}

\author{
André Martin
}

\author{
CERN, Theory Division, CH1211 Genève 23, Switzerland
}

\begin{abstract}
We explain why we hope that the Froissart bound can be improved, either qualitatively or, more likely, quantitatively, by making a better use of unitarity, in particular elastic unitarity. In other instances (Gribov's theorem) elastic unitarity played a crucial role. A preliminary requirement for this is to work with an appropriate average of the cross-section, to make the problem well defined. This is possible, without destroying the Lukaszuk-Martin bound.
\end{abstract}

Keywords: High-energy scattering, Froissart bound

PACS: $11.55 .-\mathrm{m}$

Many of my friends complain that, in the Froissart bound [1, 2] on total crosssections, the factor in front of the logarithm square is much too big. One of them is Peter Landshoff [3] present at this conference. Indeed the bound that we obtained with the late Lesek Lukaszuk [4]

$$
\sigma_{\text {total }}<\left(\frac{\pi}{m_{\pi}}\right)^{2}(\ln s)^{2},
$$

is perhaps 500 times larger than what is found in fits of the proton-(anti) proton experimental cross-sections [5]. People should remember that this is only a bound in which the mass of the pion appears because the two-pion threshold is the first one appearing in the $t$-channel. However, we believe that there is some hope for improvement. "We" means Joachim Kupsch, Jean-Marc Richard, Shasanka Roy and me. The reason is that so far there is no example of amplitude which has the right analyticity properties in all channels and satisfies "unitarity", which means for the partial wave amplitudes

$$
\begin{aligned}
& \mathfrak{I m} f_{\ell} \geq\left|f_{\ell}\right|^{2} \quad \text { for all energies, } \\
& \mathfrak{I m} f_{\ell}=\left|f_{\ell}\right|^{2} \quad \text { in the elastic region, }
\end{aligned}
$$

with

$$
\begin{aligned}
& F(s, \cos \theta)=\frac{\sqrt{s}}{2 k} \sum_{\ell}(2 \ell+1) P_{\ell}(\cos \theta) f_{\ell}(s), \\
& A(s, \cos \theta)=\frac{\sqrt{s}}{2 k} \sum_{\ell}(2 \ell+1) P_{\ell}(\cos \theta) \mathfrak{I m} f_{\ell}(s),
\end{aligned}
$$

${ }^{1}$ Dedicated to the memory of L. Lukaszuk, G. Sommer, and F.J. Yndurain

2 Talk given at "Diffraction 2008", Lalonde-les-Maures, September 2008 
where $s$ is the square of the c.m. energy, $k$ the c.m. momentum, and $\theta$ the c.m. scattering angle.

We realize that (2) and (3) represent only a small fraction of unitarity, but these are the only ingredients we can use if we don't not want to involve many-body amplitudes, much too complicated to handle.

Then, using positivity one can prove that $F$ is analytic in $t$ [6] for

$$
t<4 m_{\pi}^{2}
$$

The maximum value of $t$ in (6) is given by the prescription of Sommer, or the more refined approach of Bessis and Glaser [7], $t$ being given

$$
t=2 k^{2}(\cos \theta-1) \text {. }
$$

One can also prove that if $F$ is bounded by $s^{N}, N$ even, for $t=0$ it has also the same bound for $t=4 m_{\pi}^{2}$. Then, using unitarity, one gets the Froissart bound with a constant depending on $N$. The Froissart bound means that $N=2$ for $t=0$. Then, using the fact that the number of subtractions does not change for positive $t$, one can repeat the cycle and end up with the bound (1).

The question is whether this bound can be improved, i.e., replacing the exponent of the logarithm by an exponent smaller than 2, or quantitatively, i.e., replacing the factor in (1), that Peter dislikes, by something much smaller. Kupsch [8] has constructed an example satisfying (2) but not (3), in which the bound (1) is anyway certainly not saturated because, to simplify things, he takes the partial wave amplitudes to be less than $1 / 2$ instead of 1 . It is not even clear that (1) could be saturated using only the "inelastic" unitarity (2). However, the greatest hope for improvement comes from the fact that nobody has been able construct a model saturating the Froissart bound and satisfying both (2) ("inelastic unitarity") and (3) ("elastic unitarity"). Atkinson [9] has succeeded to construct a model satisfying (2) and (3), but his cross-section behaves like

$$
[\ln s]^{-3} \text {. }
$$

The importance of "elastic" unitarity is very well illustrated by Gribov's theorem [10] that contrary to the general belief before 1960, it is impossible to have a scattering amplitude such that

$$
F(s, t) \sim s f(t), \quad \text { with } f(0) \text { non zero },
$$

or, in words, such that the cross-section tends to a non zero constant with a finite width of the diffraction peak at infinite energy. His proof rests very heavily on "elastic" unitarity and Jean-Marc Richard and I [11] and, also, Joachim Kupsch [13], have constructed examples in which only (2) is required, and where the scattering amplitude does behave like $s f(t)$ at high energy. Our example is

$$
F=\text { Const }\left[F_{s}+F_{t}+F_{u}\right] \text {, }
$$

with

$$
F_{S}=[4-\sqrt{(4-t)(4-u)}] \exp \left[-2(4-s)^{1 / 4}\right]
$$


where $m_{\pi}=1$.

My belief is that the Froissart bound cannot be improved. May be that I am not objective because this would kill the model of my friends Claude Bourrely, Jacques Soffer, and Tai Tsun $\mathrm{Wu}[12]$. I would like to present here a sketch of an idea, which might or might not work, to construct an amplitude which saturates the Froissart bound and satisfies (2) and (3). We take the case of pion-pion scattering. We assume the validity of Mandelstam representation

$$
F(s, t)=\iint \mathrm{d} x \mathrm{~d} y \frac{\rho(x, y)}{(x-s)(y-t)}+\text { circular permutations }+ \text { substractions },
$$

with $\rho(s, t)=0$ for $s<16$ and $t<16$.

The amplitude is real for $s<16$, and we impose condition (2) only $s>16$. I believe that the construction of Kupsch can be carried completely in this framework, though I tried to have his opinion on that without success (he is trying another approach!). Anyway, suppose it works, and suppose $F$ saturates qualitatively the Froissart bound. Then, if $F$ is a solution, $\lambda F$ is also a solution, for $\lambda<1$. One can now try to correct for the fact that unitarity has not been taken into account in the elastic strip by using (3) (with $f_{\ell}$ real on the right hand side ), and obtain a first approximation of the absorptive part in the elastic region, which will be of order $\lambda^{2}$. Then one can recalculate a new amplitude using dispersion relations. This new amplitude differs from the previous one by something of the order $\lambda^{2}$. One can hope that for $\lambda$ sufficiently small a convergent iterative procedure is possible. There are difficulties in the first steps, but they might be overcome.

If this works, this means that there is no qualitative improvement possible. This would be good for our friends, Bourrely-Soffer-Wu. However, there is room for a quantitative improvement. We do not know, at the present time, how to proceed to do this. However a preliminary requirement is to work with an average of the cross-section. Among the experts of the subject, everybody knows that the Froissart bound is not local. The Froissart bound follows from the fact that the integral of $A(s, t=4)$ divided by $s^{3}$ is convergent. In fact it is possible to construct examples in which, for a given, arbitrarily high, energy the total cross-section is infinite locally. The Froissart bound applies to an average of the cross-section over an energy interval which can be very narrow, an arbitrary negative power of the energy. However the constant in the bound depends on the choice of the interval. All this was realized long ago by Common and Yndurain [14]. The constant in (1) holds for a sequence of energies with asymptotic density unity if $A(s, t=4)$ is continuous. What we have found is that if we want to avoid this assumption, and if we want this average to satisfy the bound (1) the interval over which we take the average must be of the order of $s$, for instance $s$ to $2 s$. The benefit of using this average is that the scale factor in the logarithm is fixed as a function of low-energy parameters in the $t$ channel. Therefore, another criticism to the Froissart bound is eliminated. In fact, since this talk was given, I realized that an interval of the order of $s / \ln s$, is also acceptable, which means that the interval becomes much smaller than $s$ at high energies.

Now what to do next? Honestly, we don't know. S.M. Roy would like to try a variational approach, assuming Mandelstam representation, and varying the completely inelastic part of the double spectral function, i.e., the double spectral function minus 
what is obtained from elastic unitarity in the $s$ and $t$ channels. I apologize for not being more precise! This is only a kind of progress report.

\section{Acknowledgments}

The work presented here was carried with the support of the CEFIPRA (IFCPAR), project No: 3404-3 (Indo-French collaboration). Hospitality at the I.H.E.S. ( Institut des Hautes Etudes Scientifiques, Bures sur Yvette) is also acknowledged.

\section{REFERENCES}

1. M. Froissart, Phys.Rev. 123 (1961) 1053.

2. French title by Jacques Bros, for "L'Album de la Comtesse": Peut-on reculer les bornes de Froissart ?

3. Though I heard Peter making this statement many times, in particular at the "Blois" meetings, including the first one in 1985, and also at the conferences organized by Herb Fried and his friends at the American University of Paris, I could not find any printed trace of it. I am grateful for his kindness in his writings.

4. L. Lukaszuk and A. Martin, Nuovo Cimento 52 A (1967) 122, Appendix E.

5. C. Augier et al. (UA4.2 collaboration), Phys.Lett. B315 (1993) 503.

6. A. Martin, Nuovo Cimento 42A (1966) 930.

7. G. Sommer, Nuovo Cimento 48A (1967) 92;

J.D. Bessis and V. Glaser, Nuovo Cimento 50A (1967) 568.

8. J.Kupsch, Nuovo Cimento 82A (1982) 85.

9. D. Atkinson, Nucl.Phys. B23 (1970) 397. It is amusing that in this paper David seems to be satisfied to obtain models with a slowly decreasing cross-section (like Gribov in 1960!), because this was before the experimental discovery that cross-sections are rising.

10. V.N. Gribov, Proceedings of the 1960 Annual International Conference on High Energy Physics at Rochester, Eds. E.C.G. Sudarshan, J.H. Tinlot and A.C. Melissos (University of Rochester 1960) p. 340. This communication was not presented by Gribov himself because he could not get permission from the Russians to attend the conference. It was presented, I believe, by Francis Low.

11. A. Martin and J.-M. Richard, presented by A. Martin in "Forward Physics and Luminosity Determination at LHC", Helsinki 2000, K.Huiti, V.Khoze, R.Orava, and S.Tapprogge Eds., (World Scientific 2001), p.27.

12. C. Bourrely, J. Soffer and T. T. Wu, Eur. Phys. J. C 28 (2003) 97.

13. J.Kupsch, private communication.

14. A.K. Common and F.J. Yndurain, Nucl.Phys. B26 (1971) 167 and B34 (1971) 509;

F.J. Yndurain, Phys.Lett. 31B (1970)368.

See also A.Martin in "Elastic and Diffractive Scattering", Château de Blois, June 1985, B. Nicolescu and J.Tran Than Van, Eds.( Editions Frontières 1985) p. 153. 